\title{
Dyslipidemia and Associated Factors in Tenofovir Disoproxil Fumarate-Based Regimen Among Human Immunodeficiency Virus-Infected Ethiopian Patients: A Hospital-Based Observational Prospective Cohort Study
}

This article was published in the following Dove Press journal:

Drug, Healthcare and Patient Safety

\section{Taklo Simeneh Yazie (1) \\ Pharmacology Unit and Research Team, Department of Pharmacy, College of Health Sciences, Debre Tabor University, Debre Tabor, Amhara, Ethiopia}

Background: Studies showed conflicting results regarding the effect of broadly used tenofovir disoproxil fumarate (TDF)-based regimen on lipid profiles, and in Ethiopia, there is no data regarding the magnitude of dyslipidemia and its associated factors.

Objective: The aim of this study was to determine the magnitude of dyslipidemia and its associated factors among adult human immunodeficiency virus (HIV)-infected patients in TDF-based regimen in Tikur Anbessa Specialized Hospital (TASH) in Ethiopia.

Methods: A hospital-based observational prospective cohort study was conducted on conveniently selected 63 patients in TASH from January to September, 2019. The data was analyzed by using SPSS version 21.0, and multivariate logistic regression was used to determine associated factors with dyslipidemia.

Results: The overall dyslipidemia was $73 \%$ and $77.8 \%$ at baseline and six months, respectively. The prevalence of total cholesterol (TC) $\geq 200 \mathrm{mg} / \mathrm{d}$, triglyceride (TG) $\geq 150 \mathrm{mg} / \mathrm{dL}$, low density lipoprotein cholesterol (LDL-c) $\geq 130 \mathrm{mg} / \mathrm{dL}$, and high density lipoprotein cholesterol (HDL-c) $<40 \mathrm{mg} / \mathrm{dL}$ was $38.1 \%$ vs $42.9 \%, 23.8 \%$ vs $31.7 \%, 17.5 \%$ vs $22.2 \%$, and $41.3 \%$ vs $41.3 \%$ at baseline and six month follow-up, respectively. Age $\geq 50$ years old (AOR $=0.6,95 \% \mathrm{CI}$ : $0.004-0.71, \mathrm{p}=0.026)$ and body mass index $(\mathrm{BMI}) \geq 25 \mathrm{~kg} / \mathrm{m}^{2}(\mathrm{AOR}=6.44,95 \% \mathrm{CI}: 1.34-30.9$, $\mathrm{p}=0.02$ ) were significantly associated with $\mathrm{TC} \geq 200 \mathrm{mg} / \mathrm{dL}$. Having cancer (AOR $=0.04,95 \%$ CI: $0.01-0.6, p=0.019)$ and education level below diploma $(\mathrm{AOR}=9.47,95 \% \mathrm{CI}: 1.15-77.96$, $\mathrm{p}=0.037)$ were significantly associated with overall dyslipidemia

Conclusion: In this study, the proportion of patients with dyslipidemia was higher at six month follow-up but there was no significant difference when compared to baseline. The mean LDL-c was significantly higher at six months compared to its baseline mean. The associated factors with dyslipidemia were age, BMI, having cancer and low level of education. Lipid profile monitoring is recommended in patients with a younger age and higher BMI.

Keywords: tenofovir disoproxil fumarate, dyslipidemia, associated factors, Tikur Anbessa Specialized Hospital

\section{Introduction}

Although there is no cure, antiretroviral therapies (ART) have successfully treated HIV-infected patients and transformed HIV in to a chronic medical condition. As 
considerably higher HIV-infected patients are virologically controlled and living longer, the trajectory of the disease's morbidity has shifted, however, primarily from opportunistic infections and immune dysfunction to metabolic complications. ${ }^{1}$ Metabolic abnormalities are known to increase the risk of cardiovascular disease and it occurs in association with ART, HIV itself or both. ${ }^{2}$ ART increases biosynthesis and reduces hepatic clearance of serum cholesterol thereby leading to dyslipidemia. It is evident in a systematic review that ART exposed patients had significantly higher total cholesterol (TC), low density lipoprotein cholesterol (LDL-c) and triglyceride (TG) compared to ART naïve patients. According to 25 studies, exposure to ART was associated with significantly higher rates of hypercholesterolemia compared to ART naïve patients $(27.2 \%$ of 19,967 HIV-infected patients on ART versus $9.3 \%$ of 5801 in the ART-naïve group developed hypercholesterolemia). ${ }^{3}$ A number of studies in African countries showed a high prevalence of dyslipidemia among ART exposed patients ranging from 36.9-85\%. ${ }^{4-7}$ Among nucleos(t)ide reverse transcriptase inhibitors, tenofovir disoproxil fumarate (TDF) was associated with improved lipid profiles in trials. ${ }^{8-10}$ However, in some trials and most studies in routine clinical practice, increased lipid parameters and dyslipidemia was found in a TDF-based regimen. ${ }^{11-15}$ Worldwide, including Ethiopia, TDF-based regimen is now the preferred first line ART due to its more favorable efficacy and safety compared to other ARTs. But it is not without risk of adverse effects as evidenced by studies in routine care. Nowadays, ART has been prescribed for all HIV-infected patients without delay based on the test and treat principle recommended by the World Health Organization (WHO). As a result, exposure to TDF-based regimen is increasing, which might increase the likelihood of adverse drug reaction occurrence. ${ }^{16}$

Different associated factors of dyslipidemia in HIVinfected patients are determined in studies. In HIVinfected patients, dyslipidemia is typically attributed to the adverse effects of ART. Genome wide association study validated that single nucleotide polymorphisms (SNPs) contribute to dyslipidemia both in HIV infection and ART. Both ART and the cumulative effect of SNPs contribute to the risk of high LDL-c, low high-density lipoprotein cholesterol (HDL-c) and hypertriglyceridemia. Genetic variants presumably contribute major metabolic complications such as diabetes, and coronary artery disease in HIV-infected patients. ${ }^{17}$ In two cross sectional studies, body mass index (BMI) greater than $25 \mathrm{~kg} / \mathrm{m}^{2}$, blood pressure of 140/90 $\mathrm{mmHg}$ and above, being on ART, and duration of HIV infection of 42 months and more were factors associated with dyslipidemia. ${ }^{4,5}$ Moreover, CD4 cell count greater than 500 cells $/ \mathrm{mm}^{3}$, taking ART for greater than 2 years, obese patients, and age of 50 years old and above were factors associated with dyslipidemia in another study. ${ }^{7}$ High baseline TC and high baseline TG were associated with hypercholesterolemia and hypertriglyceridemia, respectively. ${ }^{14}$ Studies recommended close monitoring of lipid profiles in patients with TDF-based regimen exposure. However, in practical patients have been taking TDF-based regimen without monitoring lipid profiles. ${ }^{13,14}$ Especially, lack of monitoring lipid profiles in TDF-based regimen is a major problem in low income countries like Ethiopia. In Ethiopia, TDF-based regimen is the preferred first line therapy and more patients are more likely to be exposed to this regimen but there is no data regarding prevalence and associated factors of dyslipidemia in the country, particularly in the study setting. Thus, this study was designed to determine magnitude of dyslipidemia and its associated factors among adult patients receiving TDF-based regimen in Tikur Anbessa Specialized Hospital (TASH).

\section{Methods}

\section{Study Design and Setting}

A hospital-based observational prospective cohort study was used to enroll adult HIV-infected patients who are going to receive a TDF-based regimen, and those treatment experienced patients whose ART are going to be switched to TDFbased regimen in TASH. TASH was opened in 1972 as a specialized hospital, and in 1998, the TASH is the largest referral hospital in the country, with 700 beds, was transferred to the School by the Federal Ministry of Health and it has since become a University Teaching Hospital. The TASH is now the main teaching hospital for both clinical and preclinical training of most disciplines. It is also an institution where specialized clinical services that are not available in other public or private institutions are rendered to the whole nation. A recent report, covering from July to October in 2018, showed that there were 3336 adult HIV positive patients and 473 pediatric patients on highly active antiretroviral therapy. The study area was selected purposively after making sure that a larger number of HIV-infected patients who were newly initiated with a TDF-based regimen were found compared to other governmental hospitals in Addis Ababa. Based on eligibility criteria, 66 participants 
were enrolled consecutively before commencing a TDFbased regimen but three participants were lost from followup after baseline visit. A total of 63 participants were followed for 6 months after commencing the TDF-based regimen and included in the final analysis. The study was conducted from January 15 to September 15, 2019 in the Gregorian calendar.

\section{Eligibility Criteria}

The study included all adult HIV-infected treatment naive patients whose ages were $\geq 18$ years old and were assigned to take a TDF-based regimen after enrollment. In addition, treatment experienced patients whose ART are going to be switched to a TDF-based regimen after enrollment were included in the study. On the other hand, participants with a TDF-based regimen previously, pregnancy, critical illness and cognitive impairment were excluded.

\section{Data Collection Instrument and Procedure}

Interviewer administered structured questionnaire was adapted from various related literatures to collect sociodemographic and clinical traits. Socio-demographic traits of the participants were sex, age, BMI, marital status, educational status, ethnicity and occupation whereas the clinical traits were a TDF-based regimen, prior exposure to ART, prior ART regimen, type 2 diabetes mellitus (T2DM), hypertension (HTN), cancer, tuberculosis, antidiabetic drugs, antihypertensive drugs, chemotherapy, proton pump inhibitors (PPI), non steroidal anti-inflammatory drugs (NSAIDs), antituberculosis drugs, cotrimoxazole prophylaxis, isoniazid prophylaxis, World Health Organization (WHO) clinical stage, CD4 count and glomerular filtration rate (GFR). In addition, like other studies, participants with baseline dyslipidemia were included in the study and the proportion of dyslipidemia at baseline and six months was compared. ${ }^{15}$

The questionnaire was written in English and translated to Amharic afterwards. The questionnaire was retranslated to English by an expert to test its consistency. Two senior nurses and laboratory technologists took a half day training regarding the objective, methodology and ethical issue of the study. The nurses collected data by using a structured questionnaire and checklist whereas laboratory technologists determined lipid parameters. A checklist was used to record the previous ART and baseline CD4 count of patients who had prior ART exposure from the patient chart. For treatment naïve patients' baseline CD4 count was done in routine care and it was recorded in the checklist. Weight and height were measured by using Seca 761 weight scales and height ruler (with meter reading) which is attached with it, respectively (made in Germany). Body mass index of participants was calculated as follows: Body mass index (BMI) $=$ weight $($ in $\mathrm{kg}) \div(\text { height }(\text { in } \mathrm{m}))^{2}$. BMI was categorized into $<25$ and $\geq 25 \mathrm{~kg} / \mathrm{m}^{2}$ to see its association with dyslipidemia based on previous literatures. 5,15

Lipid parameters were determined at baseline and 24 weeks based on previous study. ${ }^{18,19}$ Six to ten $\mathrm{mL}$ of blood sample was collected by venipuncture from 8-12 hours fasting individuals using a vacuum tube (Becton Dickinson, SA) and serum was separated within one hour of blood collection. TC, HDL-c, LDL-c and TG were measured using a Hitachi Automatic Analyzer 7600 (Hitachi, Ltd., Tokyo, Japan) at TASH, clinical chemistry laboratory. TG and TC were evaluated with enzymatic colorimetric method and HDL-c and LDL-c were analyzed by homogenous enzymatic colorimetric method. CD4+ lymphocyte count was determined using flow cytometer (FACSC alibur, Becton Dickinson, CA, USA).

In accordance with the US National Cholesterol Education Program, Adult Treatment Panel III (NCEPATP III) guidelines, an abnormal lipid profile was defined as $\mathrm{TC} \geq 200 \mathrm{mg} / \mathrm{dl}$, HDL-c $<40 \mathrm{mg} / \mathrm{dl}$, LDL-c $\geq 130 \mathrm{mg} / \mathrm{dl}$, $\mathrm{TG} \geq 150 \mathrm{mg} / \mathrm{dl}, \mathrm{TC} / \mathrm{HDL}-\mathrm{c}$ ratio $\geq 5 .{ }^{20}$ For the purposes of the study, an elevation of any one of the lipid parameters (TC, HDL-c, LDL-c and TG) which fulfilled the defined limits was considered as dyslipidemia. Overall dyslipidemia is the proportion of participants with one or more forms of abnormality in lipid profiles ( $\mathrm{TC} \geq 200 \mathrm{mg} / \mathrm{dl}$, HDL-c $<40 \mathrm{mg} / \mathrm{dl}$, LDL-c $\geq 130 \mathrm{mg} / \mathrm{dl}$ and TG $\geq 150 \mathrm{mg} / \mathrm{dl}$ ).

\section{Data Processing and Analysis}

The data collected were marked, entered, and cleaned using EPI-Data version 3.02 and SPSS version 21. For review, the data was exported to SPSS package version 21. Descriptive statistics such as frequency, percentage, median and mean with standard deviations were used to describe variables. Paired $t$-test and McNemar's test were used to compare mean values and proportion of dyslipidemia of the six month visit from baseline, respectively. Multivariate binary logistic regression analysis was done for variables with a $\mathrm{P}$ value of $<0.25$ in univariate analysis to identify independently associated factors of dyslipidemia. Statistical significance was set at $\mathrm{p}<0.05$. 


\section{Ethical Consideration}

Ethical clearance and approval of the study protocols have been received from the Addis Ababa University, School of pharmacy Ethical Review Board (Ref. No. ERB/SOP/07/ 09/2018). Ethical approval was obtained while I was a student of Master of Science in pharmacology in Addis Ababa University from around the end of 2015 to the beginning of 2019. As the study hospital, TASH is located in Addis Ababa University, I communicated with Addis Ababa University, School of Pharmacy Ethical Review Board in 2018 to get ethical approval for this study and the Ethical Review Board gave ethical approval for this study. Additionally, permission to conduct the research was obtained from the Internal Medicine Department of TASH, Addis Ababa University. Written informed consents were obtained from the study participants and this study was conducted in accordance with the Declaration of Helsinki.

\section{Results}

\section{Socio-Demographic Characteristics}

A total of $66 \mathrm{HIV}$-infected patients were enrolled in this study. Among them, 61 patients were naïve to antiretroviral therapy whereas 5 patients were treatment experienced whose ART are going to be switched to a TDF-based antiretroviral therapy because of adverse effects. Three treatment naïve participants were lost from follow-up due to nonadherence without having serum lipid measurements after baseline visits. Finally, 63 study participants were included in the analysis. The mean ( \pm standard deviation (SD)) age of the study participants was $39.7( \pm 10)$ and the majority of study participants $(43,68.3 \%)$ were female. Regarding education, 18 (28.6\%) and 28 (44.4\%) of the study participants attended primary and secondary education, respectively. Concerning occupation, the majority of the study participants $(34,54 \%)$ engaged in private work (Table 1).

\section{Baseline Clinical Characteristics of Study Participants}

At the initiation of tenofovir containing antiretroviral therapy, the majority of patients $(58,92.1 \%)$ were treatment naïve whereas the remaining $5(7.9 \%)$ had prior exposure to a zidovudine-based antiretroviral regimen. TDF + lamivudine + efavirenz were taken by $56(88.9 \%)$ patients. On the other hand, the remaining patients were taking a TDF + lamivudine + ritonavir boosted atazanavir regimen. Median
Table I Baseline Socio-Demographic Characteristics of Study Participants in Tikur Anbessa Specialized Hospital (TASH), in $2019[n=63]$

\begin{tabular}{|l|l|l|}
\hline Variables & Category & Frequency (\%) \\
\hline Sex & Female & $43(68.3)$ \\
\hline Age & $<50$ years old & $54(85.7)$ \\
\hline $\begin{array}{l}\text { Body mass index } \\
\text { (in } \mathrm{kg} / \mathrm{m}^{2} \text { ) }\end{array}$ & $<25$ & $43(68.3)$ \\
\hline Marital status & Single & \\
& Married & $8(12.7)$ \\
& Divorced & $40(63.5)$ \\
& Widowed & $11(17.5)$ \\
& Illiterate & $(6.4)$ \\
\hline \multirow{2}{*}{ Educational status } & Primary & $5(7.9)$ \\
& Secondary & $18(28.6)$ \\
& Diploma & $5(74.4)$ \\
& Degree & $7(11.1)$ \\
\hline \multirow{2}{*}{ Ethnicity } & Oromo & $20(31.7)$ \\
& Amhara & $22(34.9)$ \\
& Tigre & $6(9.5)$ \\
& Others & $15(23.8)$ \\
\hline & Governmental employee & $12(19)$ \\
& Private & $34(54)$ \\
& Not employed & $17(27)$ \\
\hline
\end{tabular}

Note: Other ethnic groups include mixed ethnicity, Gurage, Silte, Hadiya, Welayta, and Harari.

(interquartile range (IQR)) CD4 count was 241 (106-457), and $42(66.7 \%)$ patients were found to be in WHO clinical stage I.

Twenty $(31.8 \%)$ of study participants were with comorbidity. From these co-morbidities, hypertension, type 2 diabetes mellitus, cancer, tuberculosis, and kidney stone were $6.3 \%, 3.2 \%, 12.7 \%, 6.3 \%$, and $3.2 \%$, respectively. Opportunistic infections other than tuberculosis were found in $8(12.7 \%)$ participants.

Among study participants, 8 (12.7\%) were taking isoniazid prophylaxis. In addition, angiotensin converting enzyme inhibitor plus hydrochlorothiazide, and metformin plus glibenclamide were taken by 4 (6.3\%) and $2(3.2 \%)$ study participants, respectively (Table 2 ).

\section{Prevalence of Dyslipidemia Among Study Participants}

In this study, overall dyslipidemia of the study participants were $46(73 \%)$ and $49(77.8 \%)$ at baseline and six month visit, respectively. Concerning each lipid's parameters, 
Table 2 Baseline Clinical Characteristics of Study Participants in TASH, in 2019 [ $n=63]$

\begin{tabular}{|c|c|c|}
\hline Variables & Category & $\begin{array}{l}\text { Frequency } \\
\text { (\%) }\end{array}$ \\
\hline WHO stage & $\begin{array}{l}\text { I } \\
\text { II } \\
\text { III } \\
\text { IV }\end{array}$ & $\begin{array}{l}42(66.7) \\
3(4.8) \\
6(9.5) \\
12(19)\end{array}$ \\
\hline Cotrimoxazole prophylaxis & Yes & $42(66.7)$ \\
\hline Cancer & Yes & $8(12.7)$ \\
\hline Antituberculosis drugs & Yes & $4(6.3)$ \\
\hline NSAIDs & Yes & $6(9.5)$ \\
\hline Proton pump inhibitor & Yes & $9(14.3)$ \\
\hline Chemotherapy & Yes & $4(6.3)$ \\
\hline Baseline CD4 count (cells/mm3) & $<200$ & $29(46)$ \\
\hline Median (IQR) & $\begin{array}{l}241 \\
(106-457)\end{array}$ & - \\
\hline $\begin{array}{l}\text { Baseline eGFR }\left(\mathrm{mL} / \mathrm{min} / 1.73 \mathrm{~m}^{2}\right) \\
\text { Mean (+SD) }\end{array}$ & $\begin{array}{l}<90 \\
90.8(+16.8)\end{array}$ & $\begin{array}{l}35(55.6) \\
-\end{array}$ \\
\hline
\end{tabular}

Abbreviations: WHO, World Health Organization; DX, diagnosis; IQR, interquartile range; SD, standard deviation; NSAIDs, nonsteroidal anti-inflammatory drugs; eGFR, estimated glomerular filtration rate.

hypercholesterolemia, hypertriglyceridemia, raised LDL-c and decreased HDL-c were 24 (38.1\%), 15 (23.8\%), 11 (17.5\%), and $26(41.3 \%)$ at baseline, and 27 (42.9\%), 20 (31.7\%), $14(22.2 \%)$, and $26(41.3 \%)$ at six month visits, respectively. Among participants with dyslipidemia at baseline, $10(21.7 \%)$ improved and reached the physiological values. However, 13 (76.4\%) participants with no dyslipidemia at baseline developed dyslipidemia by the six month visit. From overall dyslipidemia at the end of follow-up, 7 (14.3\%) were found in participants with age of 50 years old and above. Participants who were 60 years old and above were $6(9.52 \%)$ and all were found to have dyslipidemia. However, 4 from 6 participants had dyslipidemia at baseline (Table 3).

\section{Factors Associated with Dyslipidemia}

In univariate binary logistic regression analysis, female gender, age $\geq 50$ years old, BMI $\geq 25 \mathrm{~kg} / \mathrm{m}^{2}$, and CD4 count $<200$ cells $/ \mathrm{mm}^{3}$ were significantly associated with raised $\mathrm{TC}(\mathrm{COR}=3.14,95 \% \mathrm{CI}: 0.97-10.18, \mathrm{p}=0.056$; $\mathrm{COR}=0.33,95 \% \mathrm{CI}: 0.06-1.74, \mathrm{p}=0.192 ; \mathrm{COR}=3.25$, 95\% CI:1.09-9.66, $\mathrm{p}=0.034$, and COR $=0.53,95 \%$ CI:0.19-1.46, $\mathrm{p}=0.217$ ), respectively. Age $\geq 50$ years old was significantly associated with raised $\mathrm{TG}(\mathrm{COR}=0.23$, 95\% CI: 0.03-1.98, $\mathrm{p}=0.181)$. Female gender, baseline estimated glomerular filtration rate $(\mathrm{eGFR})<90 \mathrm{~mL} / \mathrm{min}$, and CD4 count less than 200 cells $/ \mathrm{mm}^{3}$ were significantly associated with raised LDL-c (COR $=3.48,95 \%$ CI: $0.7-17.35, p=0.128$; $\mathrm{COR}=4.89$, 95\% CI: $0.99-$ 24.2, $\mathrm{p}=0.052$, and $\mathrm{COR}=0.24,95 \% \mathrm{CI}: 0.06-0.97$, $\mathrm{p}=0.046$ ), respectively. Regarding decreased HDL-c, age $\geq 50$ years old, and baseline eGFR $<90 \mathrm{~mL} / \mathrm{min}$ were significantly associated with it $(\mathrm{COR}=3.4,95 \% \mathrm{CI}$ : $0.77-15.12, \mathrm{p}=0.108$, and $\mathrm{COR}=3.16$, 95\% CI: $1.03-$ 9.65, $\mathrm{p}=0.044)$, respectively. The CD4 count of less than 200 cells $/ \mathrm{mm}^{3}$, and baseline eGFR $<90 \mathrm{~mL} / \mathrm{min}$ were significantly associated with total dyslipidemia

Table 3 Prevalence of Dyslipidemia Among Study Participants

\begin{tabular}{|l|l|l|l|}
\hline \multirow{2}{*}{ Lipid Profile } & \multicolumn{2}{|l|}{ Time of Serum Lipid Measurement } \\
\cline { 2 - 4 } & At Baseline & At Six Month Visit & p-value \\
\hline Total cholesterol, mean \pm SD & $180.21 \pm 41.502$ & $185.17 \pm 37.976$ & $0.27^{*}$ \\
$\geq 200 \mathrm{mg} / \mathrm{dL}, \mathrm{n}(\%)$ & $46(38.1 \%)$ & $49(42.9 \%)$ & $0.65^{* *}$ \\
Triglycerides, mean \pm SD & $124.37 \pm 56.802$ & $136.24 \pm 76.731$ & $0.09^{*}$ \\
$\geq 150 \mathrm{mg} / \mathrm{dL}, \mathrm{n}(\%)$ & $15(23.8 \%)$ & $20(31.7 \%)$ & $0.30^{* *}$ \\
LDL-cholesterol, mean \pm SD & $98.25 \pm 33.118$ & $108.92 \pm 34.438$ & $0.03^{*}$ \\
$\geq 130 \mathrm{mg} / \mathrm{dL}, \mathrm{n}(\%)$ & $11(17.5 \%)$ & $14(22.2 \%)$ & $0.58^{* *}$ \\
HDL-cholesterol, mean \pm SD & $43.84 \pm 13.644$ & $43.56 \pm 10.528$ & $0.85^{*}$ \\
$<40 \mathrm{mg} / \mathrm{dL}, \mathrm{n}(\%)$ & $26(41.3 \%)$ & $26(41.3 \%)$ & $1.00^{* *}$ \\
Overall Dyslipidemia, $\mathrm{n}(\%)$ & $46(73 \%)$ & $49(77.8 \%)$ & $0.52^{* *}$ \\
Total cholesterol/HDL, mean \pm SD & $4.44 \pm 1.65$ & $4.44 \pm 1.28$ & $0.99^{*}$ \\
$\geq 5, \mathrm{n}(\%)$ & $19(30.2 \%)$ & $15(23.8 \%)$ & $0.42^{* *}$ \\
\hline
\end{tabular}

Notes: Level of significance is set at $P<0.05$; ${ }^{*} P$ from $t$-test comparison of means; $* * P$ from McNemar chi square tests for paired data by time of visit.

Abbreviations: SD, standard deviation; HDL, high-density lipoprotein; LDL, low- density lipoprotein. 
Table 4 Association of Variables with Lipid Abnormalities Among Study Participants Using Univariate Binary Logistic Regression

\begin{tabular}{|c|c|c|c|c|c|c|}
\hline \multirow[t]{2}{*}{ Variables } & \multirow[t]{2}{*}{ Category } & \multicolumn{5}{|c|}{ Outcome Variables in $\mathrm{mg} / \mathrm{dl}$} \\
\hline & & $\begin{array}{l}\text { TC } \geq 200 \text { COR } \\
(95 \% \mathrm{Cl})\end{array}$ & $\begin{array}{l}\text { TG } \geq 150 \text { COR } \\
(95 \% \mathrm{Cl})\end{array}$ & $\begin{array}{l}\text { LDL-c } \geq 130 \text { COR } \\
(95 \% \mathrm{Cl})\end{array}$ & $\begin{array}{l}\text { HDL-c }<40 \text { COR } \\
(95 \% \mathrm{Cl})\end{array}$ & $\begin{array}{l}\text { Overall Dyslipidemia } \\
\text { COR }(95 \% \mathrm{Cl})\end{array}$ \\
\hline Sex & Female & $3.14(0.97,10.18)^{*}$ & $0.58(0.19,1.77)$ & $3.48(0.7,17.35)^{*}$ & $1.08(0.37,3.18)$ & $1.46(0.41,5.2)$ \\
\hline Age & $\geq 50$ years & $0.33(0.06,1.74)^{*}$ & $0.23(0.03,1.98)^{*}$ & $\mathrm{I}(0.18,5.46)$ & $3.4(0.77,15.12)^{*}$ & $0.9(0.16,4.93)$ \\
\hline $\mathrm{BMl}$ in $\mathrm{kg} / \mathrm{m}^{2}$ & $\geq 25$ & $3.25(1.09,9.66)^{*}$ & $0.8(0.26,2.5 \mathrm{I})$ & $1.7(0.5,5.76)$ & $082(0.28,2.4)$ & $1.88(0.46,7.7 \mathrm{I})$ \\
\hline $\begin{array}{l}\text { Baseline eGFR } \\
\text { in } \mathrm{mL} / \mathrm{min}\end{array}$ & $<90$ & $3.5(1.44,10.71)^{*}$ & $1.68(0.54,5.21)$ & $4.89(0.99,24.2) *$ & $3.16(1.03,9.65)^{*}$ & $8.57(2.05,35.83)^{*}$ \\
\hline Marital status & $\begin{array}{l}\text { Single } \\
\text { Married } \\
\text { Divorced } \\
\text { Windowed }\end{array}$ & $\begin{array}{l}I \\
2.71(0.49,15.1) \\
1.71(0.23,12.89) \\
3(0.24,37.67)\end{array}$ & $\begin{array}{l}\text { I } \\
0.71(0.15,3.48) \\
0.95(0.14,6.28) \\
0.56(0.4,8.09)\end{array}$ & $\begin{array}{l}\text { I } \\
2.03(0.22,18.77) \\
1.56(0.12,20.85) \\
7(0.4,123.35)^{*}\end{array}$ & $\begin{array}{l}\text { I } \\
0.14(0.03,0.81)^{*} \\
0.4(0.06,2.93) \\
0.33(0.03,4.19)\end{array}$ & \\
\hline Ethnicity & $\begin{array}{l}\text { Oromo } \\
\text { Amhara } \\
\text { Tigre } \\
\text { Others }\end{array}$ & $\begin{array}{l}0.55(0.14,2.12) \\
0.38(0.1,1.47)^{*} \\
0.13(0.01,1.44)^{*} \\
\text { I }\end{array}$ & $\begin{array}{l}\text { I.83(0.43, } 7.84) \\
\text { I.57(0.37, 6.6I) } \\
0.01(0.001,0.4) \\
\text { I }\end{array}$ & $\begin{array}{l}0.86(0.2,3.61) \\
0.2(0.03,1.22)^{*} \\
0.4(0.04,4.41) \\
\text { I }\end{array}$ & $\begin{array}{l}3.36(0.79,14.25)^{*} \\
2.75(0.67,11.34)^{*} \\
0.1(0.01,0.7) \\
\text { I }\end{array}$ & $\begin{array}{l}2.25(0.33,15.54) \\
1.13(0.21,5.95) \\
0.13(0.02,1.04)^{*} \\
\text { I }\end{array}$ \\
\hline Education & $\begin{array}{l}\text { Below } \\
\text { diploma }\end{array}$ & $2.67(0.65,11.01)^{*}$ & $2.73(0.54,13.83)^{*}$ & $1.54(0.3,8.01)$ & $4.44(0.88,22.34)^{*}$ & $6.29(1.57,25.1)^{*}$ \\
\hline WHO stage & $\begin{array}{l}\text { I+II } \\
\text { III+IV }\end{array}$ & $\begin{array}{l}\text { I } \\
0.55(0.85,1.64)\end{array}$ & $\begin{array}{l}\text { I } \\
2.91(0.96,8.85)^{*}\end{array}$ & $\begin{array}{l}1 \\
0.47(0.12,1.91)\end{array}$ & $\begin{array}{l}\mathrm{I} \\
\mathrm{I} .1(0.38,3.19)\end{array}$ & $\begin{array}{l}\text { I } \\
0.75(0.21,2.67)\end{array}$ \\
\hline CD4 & $\begin{array}{l}<200 \text { cells/ } \\
\mathrm{mm}^{3}\end{array}$ & $0.53(0.19,1.46)^{*}$ & $1.26(0.44,3.66)$ & $0.24(0.06,0.97)^{*}$ & $1.01(0.37,2.76)$ & $0.45(0.13,1.58)^{*}$ \\
\hline CART & $\begin{array}{l}\text { TDF+3TC } \\
+\mathrm{ATVr}\end{array}$ & I $(0.2,4.89)$ & $0.84(0.15,4.78)$ & $1.47(0.25,8.52)$ & $2.06(0.42,10.11)$ & $1.64(0.18,14.93)$ \\
\hline Cancer & Yes & $0.4(0.07,2.16)$ & $1.34(0.29,6.27)$ & $0.46(0.05,4.11)$ & $0.43(0.08,2.33)$ & $0.2(0.04,0.93)^{*}$ \\
\hline HTN & Yes & $4.38(0.43,44.61)^{*}$ & $0.7(0.07,7.2)$ & $3.92(0.5,30.73) *$ & $1.46(0.19,11.08)$ & \\
\hline OPI & Yes & $1.39(0.32,6.15)$ & $4.44(0.94,20.93)^{*}$ & $0.1(0.01,0.7)$ & $0.84(0.18,3.85)$ & $0.75(0.13,4.24)$ \\
\hline Chemotherapy & Yes & $0.42(0.04,4.3 \mathrm{I})$ & $2.28(0.3,17.46)$ & $1.18(0.11,12.31)$ & $1.46(0.19,11.08)$ & $0.77(0.07,8.03)$ \\
\hline PPI & Yes & I.82 $(0.44,7.54)$ & $1.09(0.24,4.88)$ & $1.96(0.42,9.08)$ & $0.67(0.15,2.98)$ & $0.9(0.16,4.93)$ \\
\hline $\begin{array}{l}\text { Cotrimoxazole } \\
\text { px }\end{array}$ & Yes & $0.56(0.19,1.61)$ & $0.65(0.22,1.97)$ & $0.27(0.08,0.93)^{*}$ & $1.22(0.42,3.56)$ & $0.53(0.13,2.19)$ \\
\hline $\mathrm{INH}$ px & Yes & $1.39(0.32,6.15)$ & I.34(0.29, 6.27) & $2.4(0.5,11.61)$ & $0.84(0.18,3.85)$ & $1.95(0.22,17.47)$ \\
\hline
\end{tabular}

Notes: Level of significance is set at $p<0.25$; *stands for significant variables.

Abbreviations: BMI, body mass index; eGFR, estimated glomerular filtration rate; WHO, World Health Organization, cART, combination antiretroviral therapy; HTN, hypertension; OPI, opportunistic infection; PPI, proton pump inhibitor; TDF, tenofovir disoproxil fumarate; 3TC, lamivudine; ATVr, ritonavir boosted atazanavir; INH px, isoniazid prophylaxis.

$(\mathrm{COR}=0.45,95 \% \mathrm{CI}: 0.13-1.58, \mathrm{p}=0.214$, and $\mathrm{COR}=$ 8.57, 95\% CI: 2.05-35.83, $\mathrm{p}=0.003$ ), respectively (Table 4).

In multivariate binary logistic regression analysis, age $\geq 50$ years old, and $\mathrm{BMI} \geq 25 \mathrm{~kg} / \mathrm{m}^{2}$ were significantly associated with raised TC (AOR $=0.6,95 \%$ CI: $0.004-0.71, \mathrm{p}=$ 0.026 , and AOR $=6.44,95 \%$ CI: $1.34-30.9, \mathrm{p}=0.02)$, respectively. Being married was significantly associated with decreased HDL-c (AOR $=0.5,95 \%$ CI: 0.006-0.44, $\mathrm{p}=0.007)$. Concerning overall dyslipidemia, having cancer and a level of education below diploma were significantly associated with it $(\mathrm{AOR}=0.04,95 \% \mathrm{CI}: 0.01-0.6, \mathrm{p}=$ 0.019 , and $\mathrm{AOR}=9.47,95 \% \mathrm{CI}: 1.15-77.96, \mathrm{p}=0.037$ ), respectively (Table 5). 
Table 5 Multivariate Binary Logistic Regression Analysis for Variables Associated with Lipid Abnormality

\begin{tabular}{|c|c|c|c|c|c|c|}
\hline \multirow[t]{2}{*}{ Variables } & \multirow[t]{2}{*}{ Category } & \multicolumn{5}{|c|}{ Outcome Variable in $\mathrm{mg} / \mathrm{dl}$} \\
\hline & & $\begin{array}{l}\text { TC } \geq 200 \text { COR } \\
(95 \% \mathrm{Cl})\end{array}$ & $\begin{array}{l}\text { TG } \geq I 50 \text { COR } \\
(95 \% \mathrm{Cl})\end{array}$ & $\begin{array}{l}\text { LDL-c } \geq 130 \\
\text { COR }(95 \% \mathrm{Cl})\end{array}$ & $\begin{array}{l}\text { HDL-c }<40 \\
\text { COR }(95 \% \mathrm{Cl})\end{array}$ & $\begin{array}{l}\text { Overall Dyslipidemia } \\
\text { COR }(95 \% \mathrm{Cl})\end{array}$ \\
\hline Sex & Female & $1.17(0.27,5.06)$ & & $1.25(0.18,8.83)$ & & \\
\hline Age & $\geq 50$ years & $0.6(0.004,0.7 \mathrm{I})^{*}$ & $0.26(0.029,2.35)$ & & $4.3 \mathrm{I}(0.74,25.09)$ & \\
\hline $\mathrm{BMI}$ in $\mathrm{kg} / \mathrm{m}^{2}$ & $\geq 25$ & $6.44(1.34,30.9)^{*}$ & & & & \\
\hline $\begin{array}{l}\text { Baseline eGFR } \\
\text { in } \mathrm{mL} / \mathrm{min}\end{array}$ & $<90$ & $2.8(0.61,12.84)$ & & $4.86(0.56,42.62)$ & $2.42(0.43,13.6)$ & $4.93(0.78,30.97)$ \\
\hline Marital status & $\begin{array}{l}\text { Single } \\
\text { Married } \\
\text { Divorced } \\
\text { Windowed }\end{array}$ & & & $\begin{array}{l}\text { I } \\
0.66(0.05,9.34) \\
0.45(0.023,8.7) \\
\text { II.02(0.37, 331.85) }\end{array}$ & $\begin{array}{l}\text { I } \\
0.5(0.006,0.44)^{*} \\
0.2(0.02,2.49) \\
0.28(0.008,9.72)\end{array}$ & \\
\hline Ethnicity & $\begin{array}{l}\text { Oromo } \\
\text { Amhara } \\
\text { Tigre } \\
\text { Others }\end{array}$ & $\begin{array}{l}0.61(0.11,3.33) \\
0.57(0.11,2.94) \\
0.15(0.008,2.95) \\
1\end{array}$ & & $\begin{array}{l}0.56(0.09,3.47) \\
0.19(0.02,1.76) \\
0.36(0.02,5.42) \\
\text { । }\end{array}$ & $\begin{array}{l}5.23(0.89,30.72) \\
3.07(0.53,18.01) \\
0.01(0.001,0.7) \\
1\end{array}$ & $\begin{array}{l}6.81(0.36,128.92) \\
2.56(0.22,29.93) \\
0.13(0.01,2.19) \\
1\end{array}$ \\
\hline Education & $\begin{array}{l}\text { Below } \\
\text { diploma }\end{array}$ & $2.17(0.29,16.12)$ & $2.6 \mathrm{I}(0.48,14.2 \mathrm{I})$ & & $4.86(0.42,56.26)$ & $9.47(1.15,77.96)^{*}$ \\
\hline WHO stage & $\begin{array}{l}\text { I+II } \\
\text { III+IV }\end{array}$ & & I.88(0.53, 6.7I) & & & \\
\hline CD4 & $\begin{array}{l}<200 \text { cells/ } \\
\mathrm{m}^{3}\end{array}$ & $0.89(0.24,3.39)$ & & $0.15(0.02,1.48)$ & & $0.28(0.04,2.13)$ \\
\hline Cancer & Yes & & & & & $0.04(0.01,0.6)^{*}$ \\
\hline HTN & Yes & $\begin{array}{l}22.54(0.53 \\
959.39)\end{array}$ & & $0.76(0.54,10.62)$ & & \\
\hline OPI & Yes & $2.53(0.45,14.36)$ & & & & \\
\hline Cotrimoxazole & Yes & & & $1.31(0.18,9.57)$ & & \\
\hline
\end{tabular}

Notes: Level of significance is set at $\mathrm{p}<0.05$; *stands for significant variables in multivariate analysis.

Abbreviations: BMI, body mass index; eGFR, estimated glomerular filtration rate; WHO, World Health Organization; Hx, history; OPI, opportunistic infection; TDF, tenofovir disoproxil fumarate; 3TC, lamivudine; ATVr, ritonavir boosted atazanavir; HTN, hypertension.

\section{Discussion}

The present study was conducted in TASH to assess dyslipidemia and associated factors among HIV-infected patients with a TDF-based regimen. The study showed that $77.8 \%$ of overall dyslipidemia was found and among patients with dyslipidemia, $71.4 \%$ were females. From participants with dyslipidemia, $71.4 \%$ of participants had two or more forms of dyslipidemia. The magnitude of dyslipidemia in the current study is consistent with other research by Dai et al of $79.3 \% .{ }^{15}$ However, this finding is higher than the finding of the study done in Zimbabwe of $64.9 \%$. This disparity of results might be due to the difference in proportion of male and female, duration of follow-up, definition of dyslipidemia, ${ }^{21}$ genetics and life style of participants. ${ }^{22,23}$

Concerning TC, the present study showed $42.9 \%$ had raised TC, higher than the findings of other studies done in China, $19.3 \%,{ }^{14}$ and Zimbabwe, $15.9 \% .{ }^{21}$ In a study in China, there was $96.6 \%$ male participants, lower baseline TC (153.52 mg/dL), lower baseline raised TC (6.5\%), and 24 months follow-up whereas the Ethiopian patients had $31.7 \%$ male participants, higher baseline TC $(180 \mathrm{mg} / \mathrm{dL})$, higher baseline raised TC (38.1\%) and 6 months followup. ${ }^{14}$ Thus, the difference of the findings might be attributed at least in part to these differences. In contrast, it is lower than the findings of other studies done in China of 
$50 \%$ and $55.1 \%$. Disagreement of the results might due to difference in proportion of male participants $(97.7 \%$ in China versus $31.7 \%$ in Ethiopia), and duration of followup (12 months in China versus 6 months in Ethiopia) between the studies, ${ }^{13,15}$ in addition to difference in life styles and genetics. ${ }^{22,23}$ The magnitude of raised TG was higher than the result of a study done in China $21.43 \%$. $^{13}$ However, it was lower than the results of other studies conducted in China $43.2 \%, 63.9 \% .{ }^{14,15} \mathrm{~A}$ difference in baseline raised TG, proportion of male participants, and duration of follow-up might be the reason in part for this disparity of results. Regarding raised LDL-c, it was $22.2 \%$ in the present study which is in line with the results of the study in Zimbabwe 20.3\%, and China 21.43\%. ${ }^{13,21}$ In contrast to this, it was lower than the results of another study in China $26.4 \% .{ }^{15}$ The decreased HDL-c magnitude was $41.3 \%$ in the current study which is in line with, lower, and higher than the findings of the studies done in Italy $40 \%$, Zimbabwe $46.6 \%$, and China $7.14 \%$, respectively. ${ }^{11,13,21}$ Variability in socio-demographic characteristics and duration of follow-up may contribute to the variation of raised LDL-c and decreased HDL-c among studies.

In the current study, overall dyslipidemia was increased from $73 \%$ at baseline to $77.8 \%$ at the six month visit, but the difference was not significant. This finding is in contrast to the result of the study done in Zimbabwe which showed a significant drop in prevalence of dyslipidemia at nine month visit compared to baseline prevalence. ${ }^{21}$ Genetics and life style variability may contribute for the disagreement of these findings. ${ }^{17,23}$ The proportion of participants with raised TC, TG, and LDL-c was higher at the six month visit than at baseline, but the increment was not significant. In line with the present study, a study done in China showed an increased proportion of participants with raised TC, TG, and LDL-c compared to baseline, but there was no significant difference from baseline. ${ }^{13}$ On the contrary to the present study, a study done in Spain showed significant decrease in proportion of participants with raised TC, and LDL-c compared to baseline whereas another study done in China revealed a significant increase compared to baseline in the proportion of participants with raised TC and TG. ${ }^{9,14}$ Regarding the magnitude of decreased HDL-c in the current study, it was $41.3 \%$, and it was the same with the baseline prevalence. This finding was in contrast to the result of a study conducted in Italy which revealed a decreased proportion of participants with decreased HDL-c, but the significance was not explained. ${ }^{11}$ Another study in China showed a significant decrease in prevalence of decreased HDL-c compared to baseline. ${ }^{13}$ The difference of lipid profiles among studies might be due to a difference in sociodemographic characteristics, life style, co-morbidity and genetics. Elevated TC/HDL-c ratio was found to be decreased from $30.2 \%$ at baseline to $23.8 \%$ at the six month visit, but this difference was not significant. Similarly, a study done in Zimbabwe showed a decreased proportion of participants with elevated TC/HDL-c ratio over time without significant difference from baseline. ${ }^{21}$

Concerning mean change of lipid profiles, there was no significant mean change in the current study except LDL-c. This finding was not in line with the result of a study in China that showed a small increase but significant in TC and HDL-c. Although the over time change of TG in a study of China is similar with the present study, LDL-c was not changed over time. ${ }^{13}$ Moreover, to the contrary of the present study, another study in China revealed a significant increase of TC and TG from baseline. ${ }^{14}$ The mean change of TC/HDL-c ratio from baseline was not significant in the current study and it is in line with the finding of a study in Italy. ${ }^{11}$ In general, lipid profiles in TDF-based regimen were variable among different studies, even among individuals within a one sample population. In the present study, some patients with dyslipidemia improved and reached physiological values while some others developed new dyslipidemia during the TDF-based therapy. Moreover, in longitudinal studies, the magnitude of dyslipidemia was found to be significantly decreased when a TDF-based regimen was given to patients with dyslipidemia. ${ }^{8,9}$ Likewise, dyslipidemia was decreased significantly from baseline among HIVinfected patients receiving a TDF-based regimen, ${ }^{21}$ and mean TC and LDL-c were decreased a small amount but significantly in male healthy volunteers receiving TDF over 4 weeks. ${ }^{10}$ In contrast to these findings, one retrospective cohort, and another prospective cohort study in China revealed a significant increase of dyslipidemia in HIVinfected patients receiving a TDF-based regimen. Another trial in China found increments in magnitude of raised TC, TG, and LDL-c, but it had not reached statistical significance which is similar to the present study. In addition, in TDF-based regimen, there was more significant increase in TC and TG compared to zidovudine (AZT)-based regimen in Cameroon. ${ }^{5}$ Similarly, a study in TASH revealed that patients in TDF-based regimen were 6.7, 1.5, and 1.9 times more likely to has raised TG, LDL-c, and TC than stavudine (D4T)-based regimen, respectively. ${ }^{24}$ The variability of findings even among participants in the same 
sample population might be due to the difference in single neucleotide polymorphisms (SNPs), life style, comorbidity, and socio-demographic characteristics among individuals and/or populations. Because SNPs were found to be associated with dyslipidemia and like ART, they contribute for the development of dyslipidemia. ${ }^{22,23,25}$

Dyslipidemia was found to be statistically associated with age, BMI, being married, having cancer, and below diploma education level. In this study an age $\geq 50$ years old was found to be less likely to have a raised TC compared to age $<50$ years old counterparts. This finding contradicts a study done by Ombeni et al. ${ }^{7}$ The variation might be due to the difference in age distribution between the studies. Studies conducted by Wilson et al and Feng et al showed that TC increases in younger age and declines in older age, which supports the present study. ${ }^{26,27}$ BMI $\geq 25 \mathrm{~kg} / \mathrm{m}^{2}$ was significantly associated with a raised TC which is consistent with a study done by Ombeni et al and another study conducted by Nsapha et al revealed that obese patients had higher mean values of raised TC compared to the nonobese patients. ${ }^{5,7}$ In the present study, being married was less likely to have decreased HDL-c, which is supported by a study done by Atiku et al. ${ }^{28}$

Education level of below diploma was significantly associated with overall dyslipidemia. This finding is supported by previous studies by Benetou et al which showed an inverse association between education level and TC values, and by Sun et al that revealed an inverse association between education level and dyslipidemia. ${ }^{29,30}$ Patients with cancer were less likely to have dyslipidemia in the current study. Contradictory results were found between studies regarding the effect of cancer on lipid profiles. There was an increase in TG, TC and LDL-c, and a decrease in HDL-c in breast cancer patients when compared with normal subjects. ${ }^{31}$ On the other hand, significantly lower TC was found in breast cancer patients compared to normal subjects. ${ }^{32}$ In addition, TC, TG, LDL-c and HDL-c were significantly lower in breast cancer patients compared to normal controls. ${ }^{33}$ In ovarian cancer patients, there was a decrease in TG, HDL-c, TC, and LDL-c when compared with normal subjects. ${ }^{31}$ Moreover, a significant decrease in values of HDL-c, TG, and TC was observed in the oral cancer patients when compared to the controls. The decrease in lipid profile in cancer patients might be due to their increased utilization of lipids by neoplastic cells in membrane biogenesis. ${ }^{34}$

The proportion of participants with dyslipidemia was increased at the six month visit but it was not significant when compared to baseline. There was a significant increase in mean LDL-c at the six month visit compared to its baseline mean value. In the future, a longitudinal study with a large sample size is recommended to identify more associated factors including SNPs and to determine the prevalence of dyslipidemia in TDF-based regimen.

\section{Limitation of the Study}

The drawback of this study is its small sample size; it may be challenging to generalize the magnitude of dyslipidemia to the general population and to identify any possible associated factors. The laboratory determination of the lipid profiles was at baseline and at the six month visit which may be limited to be able to show the trends of lipid profiles over time. Therefore, a longitudinal study with a large sample size and long duration of follow-up is required to show the trends of lipid profiles over time, prevalence of dyslipidemia and to identify more associated factors, including SNPs.

\section{Conclusion}

There was no significant difference in the proportion of participants with dyslipidemia and mean lipid profiles between six months and baseline values except mean LDL-c significantly increased over time. Higher BMI, age $\geq 50$ years old and low level of education were associated with dyslipidemia. Monitoring lipid profiles in patients with higher BMI and a younger age is recommended. In addition, education is recommended to create patients' awareness on life style changes to improve lipid profiles.

\section{Abbreviations}

ART, antiretroviral therapy; BMI, body mass index; CD4, cluster of differentiation 4; HIV, human immunodeficiency virus; TDF, tenofovir disoproxil fumarate; TASH, Tikur Anbessa Specialized Hospital; SNPs, single nucleotide polymorphisms; SPSS, Statistical Package For Social Sciences.

\section{Data Sharing Statement}

All the data generated during this study are presented in this paper. The primary raw data will be made available to interested researchers by the corresponding author if requested.

\section{Ethics and Consent}

This research was approved by the Ethical Review Board (Ref. No. ERB/SOP/07/09/2018) of School of Pharmacy, Addis Ababa University. This work was conducted in accordance with the Helsinki Declaration. Written 
informed consent was taken from each participant in this study. Data regarding personal information were coded and kept confidential.

\section{Acknowledgments}

I would like to express my deepest gratitude to all staff members of TASH ART clinic and laboratory department for their unlimited support. I would also like to extend our sincere gratitude to the data collectors for their support throughout the study period.

\section{Funding}

The author received no specific funding for this work.

\section{Disclosure}

The author reports no conflict of interest in this work.

\section{References}

1. Srinivasa S, Grinspoon SK. Metabolic and body composition effects of newer antiretrovirals in HIV-infected patients. Eur $J$ Endocrinol. 2014;170(5):R185-202. doi:10.1530/EJE-13-0967

2. Grunfeld C. Dyslipidemia and its treatment in HIV infection. Top HIV Med. 2010;18(3):112.

3. Nduka C, Sarki A, Uthman O, Stranges S. Impact of antiretroviral therapy on serum lipoprotein levels and dyslipidemias: a systematic review and meta-analysis. Int $J$ Cardiol. 2015;199(199):307-318. doi:10.1016/j.ijcard.2015.07.052

4. Bayenes HW. Prevalence and Predictors of dyslipidemia on HAART and HAART naive HIV positive persons in defense hospital, Addis Ababa, Ethiopia. Am J Health Res. 2014;2(5):303. doi:10.11648/j. ajhr.20140205.23

5. Nsagha DS, Weledji EP, Assob NJ, et al. Highly active antiretroviral therapy and dyslipidemia in people living with HIV/AIDS in Fako Division, South West Region of Cameroon. BMC Cardiovasc Disord. 2015;15(1):95. doi:10.1186/s12872-015-0090-5

6. Dave JA, Levitt NS, Ross IL, Lacerda M, Maartens G, Blom D. Antiretroviral therapy increases the prevalence of dyslipidemia in South African HIV-infected patients. PLoS One. 2016;11(3):e0151911. doi:10.1371/journal.pone. 0151911

7. Ombeni W, Kamuhabwa AR. Lipid profile in HIV-infected patients using first-line antiretroviral drugs. J Int Assoc Provid AIDS Care. 2016;15(2):164-171. doi:10.1177/2325957415614642

8. Tungsiripat M, Kitch D, Glesby MJ, et al. A pilot study to determine the impact on dyslipidemia of adding tenofovir to stable background antiretroviral therapy: ACTG 5206. Aids. 2010;24(11):1781-1784. doi:10.1097/QAD.0b013e32833ad8b4

9. Santos JR, Saumoy M, Curran A, et al. The lipid-lowering effect of tenofovir/emtricitabine: a randomized, crossover, double-blind, placebo-controlled trial. Clin Infect Dis. 2015;61(3):403-408. doi:10.1093/cid/civ296

10. Randell PA, Jackson AG, Zhong L, Yale K, Moyle GJ. The effect of tenofovir disoproxil fumarate on whole-body insulin sensitivity, lipids and adipokines in healthy volunteers. Antivir Ther. 2010;15 (2):227-233. (). doi:10.3851/IMP1518

11. Gotti D, Cesana BM, Albini L, et al. Increase in standard cholesterol and large HDL particle subclasses in antiretroviral-naive patients prescribed efavirenz compared to atazanavir/ritonavir. HIV Clin Trials. 2012;13(5):245-255. doi:10.1310/hct1305-245
12. Mills A, Crofoot JG, McDonald C, et al. Tenofovir alafenamide versus tenofovir disoproxil fumarate in the first protease inhibitorbased single-tablet regimen for initial HIV-1 therapy: a randomized Phase 2 study. J Acquir Immune Defic Syndr. 2015;69(4):439-445. doi:10.1097/QAI.0000000000000618

13. Zhao B, Zhang X, Gao F, et al. Gradually increased dyslipidemia in human immunodeficiency virus infected male patients with tenofovir plus lamivudine plus efavirenz primary treatment: a 3-year follow-up study. 2020

14. Yang J, Chen J, Ji Y, et al. Lipid profile and renal safety of tenofovir disoproxil fumarate-based anti-retroviral therapy in HIV-infected Chinese patients. Int $J$ Infect Dis. 2019;83:64-71. doi:10.1016/j. ijid.2019.03.034.

15. Dai L, Su B, Liu A, et al. Adverse events in Chinese human immunodeficiency virus (HIV) patients receiving first line antiretroviral therapy. BMC Infect Dis. 2020;20(1):158. doi:10.1186/s12879-020-4878-2

16. Organization WH. Updated recommendations on first-line and second-line antiretroviral regimens and post-exposure prophylaxis and recommendations on early infant diagnosis of HIV: interim guidelines: supplement to the 2016 consolidated guidelines on the use of antiretroviral drugs for treating and preventing HIV infection. In: World Health Organization. 2018;1-82.

17. Tarr PE, Rotger M, Telenti A. Dyslipidemia in HIV-infected individuals: from pharmacogenetics to pharmacogenomics. Pharmacogenomics. 2010;11(4):587-594. doi:10.2217/pgs.10.35

18. Erlandson KM, Kitch D, Tierney C, et al. Impact of randomized antiretroviral therapy initiation on glucose metabolism: AIDS clinical trials group study A5224s. AIDS (London, England). 2014;28 (10):1451. doi:10.1097/QAD.0000000000000266

19. Glidden DV, Mulligan K, McMahan V, et al. Metabolic effects of preexposure prophylaxis with coformulated tenofovir disoproxil fumarate and emtricitabine. Clin Infect Dis. 2018;67(3):411-419. doi:10.1093/cid/ciy083

20. National Cholesterol Education Program (NCEP). Third report of the National Cholesterol Education Program (NCEP) expert panel on detection, evaluation, and treatment of high blood cholesterol in adults (adult treatment panel III) final report. Circulation. 2002;106 (25):3143-3421. doi:10.1161/circ.106.25.3143

21. Zhou DT, Nehumba D, Oktedalen O, et al. Changes in lipid profiles of $\mathrm{HIV}(+)$ adults over nine months at a harare HIV clinic: a longitudinal study. Biochem Res Int. 2016;2016:3204818. doi: $10.1155 / 2016 / 3204818$.

22. Rotger M, Bayard C, Taffe P, et al. Contribution of genome-wide significant single-nucleotide polymorphisms and antiretroviral therapy to dyslipidemia in HIV-infected individuals: a longitudinal study. Circ Cardiovasc Genet. 2009;2(6):621-628. doi:10.1161/ CIRCGENETICS.109.874412

23. Maggi P, Di Biagio A, Rusconi S, et al. Cardiovascular risk and dyslipidemia among persons living with HIV: a review. BMC Infect Dis. 2017;17(1):551. doi:10.1186/s12879-017-2626-Z

24. Belay E, Seifu D, Amogne W, Kibret K. Lipid profile derangements among human immunodeficiency virus infected adults receiving first line anti-retroviral therapy in Tikur Anbesa specialized hospital, Addis Ababa, Ethiopia: comparative cross-sectional study. J AIDS Clin Res. 2014;5:328.

25. Mahungu TW, Nair D, Smith CJ, et al. The relationships of ABCB1 $3435 \mathrm{C}>\mathrm{T}$ and CYP2B6 516G $>$ T with high-density lipoprotein cholesterol in HIV-infected patients receiving efavirenz. Clin Pharmacol Ther. 2009;86(2):204-211. doi:10.1038/clpt.2009.78

26. Wilson PW, Anderson KM, Harris T, Kannel WB, Castelli WP. Determinants of change in total cholesterol and HDL-C with age: the framingham study. $J$ Gerontol. 1994;49(6):M252-7. (). doi:10.1093/geronj/49.6.M252

27. Feng L, Nian S, Tong Z, et al. Age-related trends in lipid levels: a large-scale cross-sectional study of the general Chinese population. BMJ Open. 2020;10(3):e034226. doi:10.1136/bmjopen-2019-034226 
28. Atiku M, Yusuf A. Marital status and occupation versus serum total cholesterol and hdl-cholesterol levels in healthy adults from Kano metropolis, Nigeria. Bayero J Pure Appl Sci. 2011;4(1):110-111.

29. Benetou V, Chloptsios Y, Zavitsanos X, Karalis D, Naska A, Trichopoulou A. Total cholesterol and HDL-cholesterol in relation to socioeconomic status in a sample of 11,645 greek adults: the EPIC study in Greece. Scand J Public Health. 2000;28(4):260-265. doi:10.1177/14034948000280040801

30. Sun G-Z, Li Z, Guo L, Zhou Y, Yang H-M, Sun Y-X. High prevalence of dyslipidemia and associated risk factors among rural Chinese adults. Lipids Health Dis. 2014;13(1):189. doi:10.1186/1476-511X13-189

31. Qadir MI, Malik SA.Plasma lipid profile in gynecologic cancers. Eur J Gynaecol Oncol. 2008;29(2):158-161.
32. Shah FD, Shukla SN, Shah PM, Patel HR, Patel PS. Significance of alterations in plasma lipid profile levels in breast cancer. Integr Cancer Ther. 2008;7(1):33-41. doi:10.1177/1534735407313883

33. Li X, Liu Z-L, Wu Y-T, et al. Status of lipid and lipoprotein in female breast cancer patients at initial diagnosis and during chemotherapy. Lipids Health Dis. 2018;17(1):91. doi:10.1186/s12944-018-0745-1

34. Reddy AV, Killampalli LK, Prakash AR, Naag S, Sreenath G, Biraggari SK. Analysis of lipid profile in cancer patients, smokers, and nonsmokers. Dent Res J (Isfahan). 2016;13(6):494. doi:10.4103/ 1735-3327.197036

\section{Publish your work in this journal}

Drug, Healthcare and Patient Safety is an international, peer-reviewed open-access journal exploring patient safety issues in the healthcare continuum from diagnostic and screening interventions through to treatment, drug therapy and surgery. The journal is characterized by the rapid reporting of reviews, original research, clinical, epidemiological and post-marketing surveillance studies, risk management, health literacy and educational programs across all areas of healthcare delivery. The manuscript management system is completely online and includes a very quick and fair peer-review system. Visit http://www.dovepress.com/testimonials.php to read real quotes from published authors. 